\title{
EMPRESAS DE CONSTRUCCION COLOMBIANA CAMINO A LA COMPETITIVIDAD ${ }^{1}$
}

Artículo de Reflexión - Recibido: 12 de Agosto de 2015 - Aceptado: 15 de Noviembre de 2015

\section{Silvia Ximena Morales²}

Universidad de Manizales. Manizales, Colombia. silviaximenamorales@gmail.com

Para citar este artículo / to reference this article:

Morales, S. (2016). Empresas de construccion colombiana camino a la competitividad. Módulo Arquitectura CUC, Vol.16 N¹, 119-134.

\section{Resumen}

En el presente artículo se hace una reflexión acerca de la competitividad de las pequeñas empresas de arquitectura y construcción colombiana teniendo como objetivo primordial analizar el impacto de la globalización en estas. En este artículo hablaremos sobre algunos tipos de economía y sobre los temas que circundan tan amplio tratado, unificando diferentes conceptos que se manejan en la actualidad, de la evolución que ha tenido a través del tiempo, los impactos positivos y negativos generados en diferentes lugares del mundo y de la actualidad económica, en especial el desarrollo económico de Latinoamérica (Colombia) analizando las posibilidades de competitividad, con base a investigaciones realizadas por diferentes autores. Por lo dicho anteriormente resulta sumamente interesante indagar en los distintos aspectos de esta ciencia tan compleja que nos atrajo especialmente por su costado social, y por la posibilidad de ser una herramienta de cambio y de transformación.

\section{Palabras clave}

Competitividad, Construcción, Arquitectura, Globalización. 


\title{
COMPANIES OF COLOMBIAN CONSTRUCTION WAY TO COMPETITIVENESS
}

\begin{abstract}
In this article, a reflection is made on the competitiveness of small Colombian architectural and construction companies, with the primary objective of analyzing the impact of globalization on these. In this article we will talk about some types of economy and the issues that surround such a broad treatise, unifying different concepts that are handled today, the evolution it has had over time, the positive and negative impacts generated in different places of the economy. World and current economic situation, especially the economic development of Latin America (Colombia), analyzing the possibilities of competitiveness, based on research carried out by different authors. From what has been said above it is extremely interesting to investigate in the different aspects of this complex science that attracted us especially for its social side, and for the possibility of being a tool of change and transformation.
\end{abstract}

\section{Keywords}

Competitiveness, Construction, Architecture, Globalization 


\section{Introducción}

A la hora de hablar de economía podría venir a nuestra cabeza principalmente la pregunta ¿De qué tipo de economía hablamos? Sin embargo, hace un tiempo atrás el término solo nos refería a una ciencia exacta que mediante modelos muy complejos intentaba comprender la realidad y dar respuestas a sus problemas anticipándose a ellos. Aunque parezca paradójico dicha mirada resultaba hegemónica, pero dejaba de lado los aspectos sociales, políticos y principalmente populares de dicha disciplina, pretendiendo por tanto ser una ciencia objetiva y a semejable a las ciencias naturales. Ahora bien, al indagar en la etimología de nuestro término estrella, encontramos que proviene del griego oikos que significa "casa", y nomos, que significa "ley" o "gobierno"; interpretarlo nos brinda cierto aire acogedor que evoca la gestión de la familia y la disposición del hogar (Alcoverro, 2013). Pese a ello la economía resulta algo muchísimo menos acogedor de lo que la etimología del término parece sugerir, porque desde la aplicación de los modelos matemáticos que nos ayudan a comprender el comportamiento de los seres en ocasiones de actos económicos, la economía se ha convertido en una ciencia más parecida a las exactas que a las sociales.

\section{Antecedentes}

La Unión Soviética, oficialmente llamada Unión de Repúblicas Socialistas soviéticas, fue un Estado federal constitucionalmente socialista que existió en Europa y Asia entre 1922 y 1991. El Estado soviético fue estructurado bajo un Gobierno nacional y una economía altamente centralizados. Con la disolución de la Unión Soviética se formaron varios países como Rusia, Ucrania, etc. quienes están caminando hacia la dirección de los países capitalistas. Casi todos los países están compitiendo para lograr el desarrollo que les permita obtener ganancias. La palabra competitividad es usada en varias partes, como la competitividad del país, la competitividad de la industria, la competitividad de las empresas o la competitividad de las personas (UM, 2006).

Colombia quiere competir con varios países y actualmente se encuentra negociando varios tratados de libre comercio, aunque es importante mencionar que Colombia es un país que posee alto potencial en el sector agroindustrial que inicia el 2010 en el puesto 72 del Índice de Desempeño Logístico que elabora el Banco Mundial, 10 puestos por encima en comparación al 2007. 
Sin embargo, son muchas las metas que quedan pendientes. La economía del país reclama nuevos sistemas de logística del transporte de carga para mejorar en la competitividad y es necesario entender que no basta con los proyectos de infraestructura vial programada y por programar. La logística implica avanzar en la reducción de los costos asociados al flujo de bienes y servicios; en la articulación de los diferentes actores que participan en los procesos de intercambio, en la ampliación de la oferta de servicios logísticos y, principalmente, en la provisión de infraestructura logística especializada (Álvarez, Pabón, \& Ortiz, 2010).

En los últimos 7 años, el país ha registrado tasas de crecimiento positivas, entre los años 2007 y 2012 las exportaciones colombianas han crecido a un promedio anual de $21.2 \%$. Adicionalmente, el Gobierno Nacional, con el liderazgo del Ministerio de Comercio, Industria y Turismo, sostiene en la actualidad una amplia agenda de negociaciones comerciales con países como Canadá, China y España, y con organizaciones internacionales como la Comunidad Andina de Naciones (CAN) y la Unión Europea. Bajo este escenario y de cara a una eventual recuperación de la economía, Colombia afrontará grandes retos en materia de productividad y competitividad que la obligan a adoptar medidas de política que permitan la inserción adecuada del país en el entorno global (Quesada, 2011).

La Competitividad de Colombia ha mejorado un poco, pero debemos concentrarnos, no solo en los sectores que tienen más campo, sino en cada sector. Se dicen que Colombia está 30-60 años atrás de Japón. Pero, de acuerdo con los indicadores de IMD en el 2007, Japón es el número 17 y Colombia ocupa el rango número 40, que no significa una gran diferencia, es verdad que existe una Diferencia de la raza, Diferencia de la cultura, y Diferencia de historia, pero esto no quiere decir que exista una diferencia de muchos años entre Japón y Colombia.

Los productos, equipos, y sistemas producidos en Colombia son más o menos iguales a los japoneses, y la construcción de la sociedad que existe en Colombia no es inferior a la de otros países (UM, 2006).

Que Colombia pueda mejorar rápidamente no es gran problema, si Colombia está atrasado por unos años puede aprovechar que otros países están durmiendo, y puede crecer tan rápido como así lo quiera. 


\section{Marco Teórico}

Debemos analizar por un momento, y cuestionarnos ¿Sabemos qué es Competitividad? Para algunas personas no es claro si el significado de competitividad es ganar o es perder, es que algo sea superior o inferior con respecto a otra cosa. La definición de competitividad de la Harvard Business School consiste en la habilidad de un país para crear, producir y distribuir productos o servicios en el mercado internacional, manteniendo ganancias crecientes de sus recursos.

Cerca del año de 1830, cumplida la fase de conformación del capitalismo, que es un sistema político, social y económico en el que grandes empresas y unas pocas personas acaudaladas controlan la propiedad, incluyendo los activos capitales (Río, 1976). Después de la revolución industrial en Europa, algunos países han culminado la buena posición en los ramos industriales y comerciales, y se les ha llamado países desarrollados, así mismo existen algunos países que se encuentran avanzando en estos aspectos, llamados países en vías de desarrollo.

Esta clasificación no muestra quién es el más fuerte entre los países desarrollados y los países que están en vía de desarrollo. Porque, los países desarrollados, son aquellos que se están esforzando para lograr la prosperidad, y los países en vías de desarrollo, son los países que se esfuerzan poco a poco para lograrlo. Una de las diferencias entre un país desarrollado y uno en vías de desarrollo suele ser la cantidad de tecnología usada por su gente. Un país desarrollado cuenta con más tecnología avanzada. Es necesario tener en consideración el estándar de vida para determinar si un país está desarrollado o en vías de desarrollo. Esto puede determinarse analizando la expectativa de vida, la tasa de analfabetismo y el Producto Interno Bruto per cápita. La ONU ha creado una estadística llamada Índice de Desarrollo Humano que determina el nivel de desarrollo de un país.

No existe una definición de competitividad internacional, pero existe una cifra llamada IMD abreviación de internacional Institute for Management Development un instituto en la ciudad de Rosan de Suiza, esta organización se encarga de reportar el rango de competitividad de los países cada año a partir de 1989. Hay otra cifra que se llama cifra Dabos Conferencia, pero la cifra de IMD es más famosa y conocida como cifra de Competitividad Internacional, hasta el año de 1992, Japón fue el primero en el Mundo en 
los puntos totales, ahora lo supera Hong Kong. La competitividad internacional de una empresa deriva, por lo tanto, de la habilidad de sus dirigentes para administrar la interacción entre estos ambientes, obteniendo una participación significativa y estable en el comercio mundial de bienes y servicios. La noción de competitividad se relaciona con una participación exitosa en el mercado internacional, buena parte de los estudios tratan a la competitividad como un fenómeno directamente relacionado con las características de desempeño y eficiencia técnica de las empresas y consideran que la competitividad de las naciones resulta como una especie de agregado de esos resultados individuales. Cada vez existe mayor consenso de que un análisis de la competitividad basado solamente en aspectos como precios, costos y tasas de cambio no son insuficientes. Por ejemplo, resulta imprescindible el dominio de técnicas productivas que es, en última instancia, lo que permite a una empresa competir con éxito en el mercado internacional. En este sentido, debe enfatizarse que la capacidad de la empresa para convertir insumos en productos con el máximo rendimiento se relaciona con su habilidad para permanecer cerca de las mejores prácticas internacionales. Bajo esta perspectiva se puede definir competitividad como la capacidad de la empresa para formular y aplicar estrategias coherentes que le permita ampliar, de manera duradera, una posición significativa en el mercado (Garay, 2004). Se identifican tres grandes grupos de factores determinantes de la competitividad. Los empresariales, los estructurales y los sistémicos. Factores Empresariales: Los factores empresariales se refieren a aquellos sobre los cuales la empresa tiene poder de decisión y que puede controlar y modificar a través de su conducta. En este grupo pueden clasificarse los siguientes: Gestión: Incluye tareas administrativas típicas, tomas de decisión, mínimo tiempo en el proceso decisión y acción, finanzas, mercadeo, actividades pos venta relaciones estrechas con los clientes, capacitación y planeación estratégica. Estas tareas forman parte del conjunto de factores críticos para el éxito en un mercado específico. Innovación: Las acciones en este campo constituyen un elemento central para la competitividad, ya sea para capturar mercados, para introducir nuevos productos y procesos, para ser más productivo o para competir en precios. La importancia de la innovación tecnológica es inequívoca en la medida en que el resultado económico de la empresa está íntimamente ligado con su capacidad de generar eficientemente 
progreso técnico, en especial en el contexto internacional. Producción: Los avances tecnológicos mundiales revelan la consagración de un nuevo paradigma productivo sobre la base de la microelectrónica, robótica, sistemas digitales de control, etcétera, en el que la calidad, diversidad y renovación de los productos, la flexibilidad y rapidez de entrega y la racionalización de los costos de producción se constituyen en apalancamiento de la competitividad. Recurso humano: Contempla el conjunto de condiciones que caracterizan las relaciones de trabajo y los aspectos que influyen en la productividad, calificación y flexibilidad de la mano de obra. En este sentido, la tarea central es definir y aplicar principios de organización y operación de procesos de trabajo que mejoren continuamente la calidad de los productos y procesos. Es importante, también, motivar a los trabajadores a participar en los desafíos competitivos y hacerlos partícipes de las ganancias derivadas del aumento de eficiencia. Factores Estructurales: En el segundo grupo de factores, los estructurales, la capacidad de intervención de la empresa es limitada, puesto que ellos se refieren al tipo de mercado, a la estructura industrial y al régimen de incentivos y regulaciones propios de cada uno. Como factores estructurales se identifican los siguientes: Mercado:
Entre sus principales elementos característicos están, entre otros: tasa de crecimiento, distribución geográfica, requerimientos tecnológicos, acceso a mercados internacionales y sistemas de comercialización. Por ejemplo, al estimularse la inversión de las empresas, los mercados dinámicos aseguran una elevada tasa de renovación de equipos y métodos de producción que aunada a las economías de escala, propician crecimientos en la productividad industrial. Otro elemento que favorece la competitividad es la presencia de consumidores exigentes de altos patrones de calidad en los productos, lo cual lleva a las empresas a adoptar estrategias competitivas de capacitación y mejora continua de la eficiencia. Estructura industrial: Se refiere a las tendencias de progreso técnico, a las escales típicas de operación física, a la relación de las empresas con sus proveedores y a la relación capitaltrabajo. En la medida en que sean más importantes las nuevas fuentes de competitividad y la pérdida de importancia de las ventajas competitivas tradicionales basadas en la disponibilidad de recursos naturales y mano de obra barata, más necesarios serán ajustes diferentes a los convencionales como ocurre en el caso de las configuraciones industriales. Es la situación de las pequeñas y medianas 
empresas que buscan economías de aglomeración espacial por medio de la constitución de polos regionales de producción como un mecanismo eficiente para favorecer el acceso a equipos y servicios sofisticados, a insumos y a redes de comercialización. Incentivos y regulaciones: En general, los incentivos tratan de aumentar la capacidad de respuesta de las empresas a los desafíos impuestos por la competencia, mientras que las regulaciones buscan condicionar sus conductas en direcciones socialmente deseables. Cuando se trata la industria a nivel agregado, estas cuestiones son de naturaleza sistémica -tercer factor determinante de la competitividad-, pero sectorialmente existen ciertas especificidades diferenciales en términos del impacto de incentivos fiscales y financieros, de políticas comerciales y de leyes del Estado. Los sectores normalmente contemplados en los programas de apoyo son las industrias nacientes, las que están altamente expuestas a la competencia internacional como la automotriz y las industrias tradicionales como la textil y siderúrgica.

Factores Sistémicos: Por último, el tercer grupo de factores que afectan la competitividad son los sistémicos. Estos constituyen externalidades para la empresa y, por tanto, la posibilidad de intervenir individualmente sobre ellos es escasa o casi que ninguna.

Estos factores son de muy diverso tipo: macroeconómico, tasa de cambio,impuestos, oferta de crédito, política salarial, crecimiento del PIB, etc., políticos e institucionales, política tributaria y tarifaria, participación del Estado en la economía, legales y regulatorios, protección a la propiedad industrial, al medio ambiente, al consumidor y al capital extranjero, sociales ,políticas de educación y formación del recurso humano, seguridad social, internacionales tendencias del comercio mundial, flujos de capital, relación con organizaciones multilaterales, acuerdos internacionales y de infraestructura, disponibilidad, calidad y costo de energía, transporte, comunicaciones, servicios tecnológicos como metrología y normalización, etcétera.

Los factores empresariales y sistémicos tienen una incidencia de tipo horizontal: esto es, presentan un carácter más genérico en la forma e intensidad como afectan la competitividad de los diferentes sectores industriales. En contraste, los factores estructurales tienen un carácter sectorial específico, afectando más directamente las especificidades que presenta cada rama de la producción o grupo de sectores similares. Estas 
consideraciones generales implican que el análisis de la competitividad debe tener en cuenta simultáneamente tanto los procesos internos de la empresa y a la industria como las condiciones económicas e institucionales del ambiente productivo.

\section{Análisis}

En la actualidad casi todas las ciudades del mundo luchan por ser competitivas y aparecer en los primeros lugares de los rankings internacionales. El logro de este propósito, tiene como afán posicionarse en el circuito internacional del capital. Bajo este objetivo, la mayor parte de las políticas urbanas impulsadas, tanto por autoridades locales como regionales y nacionales, tienen como finalidad principal generar las condiciones para acelerar el crecimiento económico de las economías metropolitanas, dinamizando preferentemente actividades ligadas a la construcción, comercio y servicios financieros, bases del modelo globalizador hegemónico que ha cautivado a las autoridades metropolitanas.

Esta estrategia de posicionamiento internacional de las áreas metropolitanas, sigue siendo aplicada por algunos estados latinoamericanos, a pesar de que diversas investigaciones han dado cuenta de los riesgos para la cohesión social y la sustentabilidad ambiental que tiene esta opción que enfatiza el logro de fines económicos y subordina otros aspectos que tienen impacto significativo en la calidad de vida urbana. Esta preocupación, también ha sido abordado por organismos supranacionales como la Unión Europea, la Organisation for Economic Cooperation and Development (OECD) $\mathrm{e}$ institucionales regionales como Comisión Económica para América Latina y el Caribe (CEPAL), las cuales han puesto en duda los reales efectos de la competitividad y de las políticas de liberalización económica, sobre todo para resolver las desigualdades sociales y lacohesión social de las grandes áreas metropolitanas.

Colombia es el séptimo país en términos de competitividad en América Latina, seguido de cerca por Perú, Argentina y Ecuador. Así lo afirma un estudio realizado por el Instituto de competitividad Aden, una red educativa de negocios de origen argentino. Pese a ser considerada por muchas compañías como una de las economías más importantes y prometedoras de la región, el país se ha ubicado sistemáticamente en el séptimo puesto durante los últimos años y ha dejado que naciones más pequeñas como Chile, Panamá y Costa Rica ganen 
terreno con ritmos de desarrollo acelerados. Colombia ha venido avanzando en competitividad, pero otros países lo hacen más rápidamente y le ganan posiciones. Tanto que en el último semestre Perú se le acercó tanto que casi lo alcanza. Según la investigación, las principales razones de que el país se ubique en este puesto son sus falencias en términos de estabilidad macroeconómica y aspectos institucionales pues no se aleja demasiado de los promedios regionales. Hay algunos aspectos como cobertura de necesidades básicas, educación y acceso a la tecnología donde está por encima de los promedios, pero hay otros como estabilidad macroeconómica o factores institucionales, donde está por debajo delos promedios. Cabe aclarar que el país no se encuentra mal en ningún indicador. De acuerdo con datos del Foro Económico Mundial (WEF) y del International Institute for Management Development, (IMD), Colombia se mantiene en unos estándares que difícilmente le permitirán lograr la visión que se tiene al año 2032, de ser la tercera economía más competitiva de América Latina. De acuerdo con el profesor Michael Porter, de la Escuela de Negocios de Harvard, la competitividad se define por la productividad con la que un país utiliza sus recursos humanos, económicos y naturales
(Restrepo, 2004). Recientemente, el Premio Nobel de Economía Paul Krugman pronunciaba una frase que ha dado para que organismos como el BID desarrollen planes orientados en ese sentido. Krugman insistía en que La productividad no es todo, pero en el largo plazo es casi todo. De esta forma, parece necesario enfocarse aún más en el tema de la productividad, debido a que una adecuada dotación del entorno es una condición necesaria pero no suficiente para el logro de una mejora consistente de la productividad y, por consiguiente, de la tan necesaria competitividad.

Porter orienta aún más su teoría cuando muestra que realmente se crea riqueza a nivel microeconómico de la economía, arraigada en la sofisticación de las compañías, así como en la calidad del entorno microeconómico empresarial en el que compiten estas compañías. De otro lado, el parque empresarial colombiano muestra un panorama que debe estar monitoreado permanentemente, ya que el 99,9 por ciento de las empresas son micro, pequeñas y medianas, siendo únicamente el 0,1 por ciento la participación de las grandes empresas en cantidad (Dane, 2005). Las denominadas mipymes aportan cerca de un 80 por ciento del empleo, según la misma fuente. 
De acuerdo con datos de la Cepal (Comisión Económica para América Latina y el Caribe, 2010), la productividad de las pymes con respecto a las grandes empresas el Colombia es de un 48 por ciento, lo que significa que para lograr resultados equiparables, las pymes deben utilizar casi el doble de los recursos que las grandes empresas. Esto no sería una dificultad si se tratara de un aspecto meramente doméstico; el asunto es que la productividad de las pymes de los países desarrollados es muy superior a la nuestra, considerando entonces que, ante los ya firmados y eventuales tratados de libre comercio se debería hacer un mayor esfuerzo para lograr que nuestras empresas mejoren su productividad y, por lo tanto su competitividad en los escenarios globales.

Para finalizar, se debe resaltar el Documento Conpes 3484 (Política nacional para la transformación productiva y la promoción de las micro, pequeñas y medianas empresas: un esfuerzo público-privado), en el que se proponen estrategias para las mipymes, pero subrayando el aspecto de ser un esfuerzo públicoprivado. Como se dijo anteriormente, este tema no es algo que le competa únicamente al Estado; es un tema que debe ser abordado desde adentro de las organizaciones también, para lo cual se requiere de las competencias de la academia y de expertos que promuevan mecanismos de colaboración y de crecimiento sostenido y sostenible de la productividad y de la competitividad.

\section{Competitividad de las empresas de construcción y arquitectura en Colombia}

Las grandes y fuertes crisis que ha experimentado el sector de la construcción y las consecuencias que éstas han tenido, nos deben permitir un conocimiento mayor y convertir esas experiencias en algo positivo, transformándolas en oportunidades para mejoramiento de los procesos en construcción. Las empresas de construcción y arquitectura deben invertir en programas de gestión de calidad con la utilización de procesos claros, generando índices de productividad altos en la ejecución de proyectos de construcción. Se debe cambiar de mentalidad sobre estos procesos de calidad y que no se tomen como un gasto, sino como una inversión que favorecerá muy seguramente los resultados de la obra, ya que esto permitirá dejar beneficios económicos, tanto para el cliente como el constructor. (Moreno, 2001). 
Los procesos de competitividad permiten y obligan a las empresas de construcción y arquitectura a implementar procesos internos que certifiquen la calidad de los trabajos sin importar si es una empresa de consultoría, diseño o construcción. Así mismo se debe garantizar calidad en la ejecución de las obras y crear procesos de inspección encaminados a la productividad de nuestras obras. (Moreno, 2001)

Para tener en cuenta estas empresas como reactivadoras de la economía, es importante saber, que son generadoras de empleo, atentas a generar cambios e implementación de acciones y procesos. En Colombia, estas empresas, están regidas por el Ministerio de Comercio, Industria y Turismo; tiene como marco normativo la Ley 905 de 2004 por medio de la cual se modifica la Ley 590 de 2000 sobre promoción del desarrollo de la micro, pequeña y mediana empresa colombiana y se dictan otras disposiciones.

En la actualidad, Colombia está nuevamente en proceso de reactivación de su economía, gracias a la firma de tratados de libre comercio con diferentes países, al avance en procesos tecnológicos, al invo- lucramiento de la academia en los procesos investigativos y de desarrollo de las mipymes, a las alianzas estratégicas con otros países (vecinos en su mayoría); aspectos que permiten visualizar este tipo de empresas y generar competitividad en los negocios. Ahora bien, si tenemos en cuenta que el desarrollo endógeno se considera un modelo de desarrollo que busca potenciar las capacidades internas de una región o comunidad local, se puede evidenciar que hoy día, el desarrollo endógeno de la región ha empezado a expandirse y a tocar de una $u$ otra forma su alrededor y aunque es evidente que Colombia tiene todavía una tasa considerable de analfabetismo (6.6\% para el año 2011), el Gobierno ha implementado acciones tendientes a formar a esta población. Sin embargo, uno de los factores a atacar es la "Pobreza", hecho que aún no demuestra cambios. Tristemente la educación en el país es costosa y aquella que es gratuita es de mala calidad, puesto que los recursos son desviados y nunca llegan completos a su destino final; es por esta razón que aún hoy se habla de querer ser un país desarrollado, teniendo niños que duran 2 horas caminando para llegar a su escuela sin haber desayunado y que la alimentación que 
se provee no atiende las necesidades nutricionales para que un niño tenga un desarrollo cognitivo aceptable. Esta es la mano de obra que se contrata en muchas empresas del país, puesto que se considera que entre menos conocimiento tenga el individuo, mejor porque de esta manera, será más barato contratarlo, menos probabilidades de robo de información y demás aspectos que afecten directamente a la empresa.

\section{Conclusiones}

La competitividad de una empresa se basa en el patrón organizativo de la sociedad en su conjunto; intervienen múltiples factores que están interrelacionados, razón por la cual se considera sistémica. Calva (2007:346) la define como producto determinado por los factores institucionales y los efectos derivados de la instrumentación de políticas públicas. Las mipymes son la principal fuente de generación de empleo, apoyando los objetivos del milenio y tratando de aportar con esto a la disminución de la pobreza generando empleo y mejorando la calidad de vida de los individuos. Para que las mipymes en Colombia, puedan garantizar su permanencia y ser competitivas frente a la globalización, debe trabajarse teniendo en cuenta la cabeza de la empresa, es decir la gerencia, que es donde se toman las decisiones y quien tiene que velar por el cumplimiento de las políticas públicas, la implementación de mecanismos que permitan optimizar recursos, aumentar la productividad con calidad y servicio, y tener una visión futurista. La capacitación del personal, salarios acordes al perfil, condiciones adecuadas de trabajo, infraestructura segura y que responda a las necesidades del mercado, son fundamentales para que las mipymes entren en la era de la globalización y empiecen a competir con tecnología y procesos innovadores. Teniendo las mipymes una gran representatividad en el sector económico del país, se requiere que el desarrollo de estas de respuesta a las necesidades de un mercado globalizado, en donde la cultura y la idiosincrasia de los territorios es diferente y en donde el idioma juega un papel importante para las negociaciones. La historia es una vieja dama que siempre tiene algo nuevo para decirnos y sorprendernos. $Y$ ahora estamos sorprendidos tratando de preguntarnos qué está pasando. El colapso que vive el mundo desde hace un tiempo, va más allá del 
plano económico y abarca una crisis cultural sin precedentes en la historia reciente (Foster, 2011). Colombia va por una muy buena senda, por varias razones. Muchas de las mediciones que podemos mirar muestran cómo el país ha progresado en la dirección correcta. Desde el año 2005 a partir del cual se desarrolló una nueva estructura sobre competitividad, en la cual está integrado el sector privado, y que es admirable. Se observa que todavía existe, aún es fuerte. La filosofía del sistema central de competitividad ha hecho una transición de un gobierno a otro, algo que siempre es un punto de alto riesgo en el desarrollo económico. Debemos resaltar que hace un par de años se empezó a reenfocar en las regiones. Se entiende que hay progreso, hay esfuerzos y buenas señales pero deben organizarse, y analizar cómo trabajan. En Colombia existe una habilidad para actuar, para hacer las cosas, para tener continuidad. Se sabe que hay muchas cosas por hacer, pues la desigualdad es un reto fundamental. Pero siempre se debe ser muy optimista porque ahora la gente está trabajando en el tema a un nivel que impresiona. Hay un consenso notable (Foster, 2011). La competitividad, si se entiende debidamente, es la raíz de la prosperidad. La idea central de competitividad es productividad. Cada lugar, cada nación, tiene geográficamente algunos recursos, incluyendo a la gente que vive ahí. La forma como se vive es la forma como se aprovecha de esos recursos. Esto determina salarios y trabajos. La competitividad se convierte en la verdadera ruta de la prosperidad. El problema radica en que se piensa mayoritariamente que a la competitividad se puede llegar por diferentes caminos. $Y$ algunas de las definiciones son realmente peligrosas. Debemos apostar por la productividad. Si cada país es más productivo, quiere decir que cada país va a ser más próspero. No hay una demanda fija en el mundo, hay una demanda infinita en bienes y servicios para las necesidades humanas. Una de las cosas más importantes para un país cuando se habla de políticas económicas es tener un lenguaje común. El país está en la misma página al pensar acerca del asunto. Una de las razones por las cuales la competitividad en Colombia ha ido creciendo lentamente es porque el país es abundante en recursos y ha sido posible que la economía crezca a buen ritmo aprovechando esas ventajas. Hay muchas cosas que tienen que ser 
arregladas en Colombia. El país tenía una plataforma muy inestable por múltiples cuestiones como la seguridad, pero tiene un gran espíritu empresarial. Existen, sin embargo, problemas con las habilidades, con las leyes laborales, que hacen más difícil ser productivo. El país tiene gente que es hábil, que persevera, que persiste y lo ha demostrado siempre. A medida que el tema de la seguridad se ha estabilizado, ha habido un aumento de la inversión extranjera. Hace 20 años se creía por muchas razones que países como India y China fueran a mejorar. Si volvemos dos décadas atrás, se pensaba que Japón era el modelo económico del futuro. En el mundo se repitieron experiencias japonesas porque fueron exitosos, pero nadie miró por qué fue creado ese modelo y cuáles eran los problemas de ese sistema. En la actualidad ha habido cambios sociales en la mayoría de países desarrollados. El movimiento ambientalista ha tenido mucho que ver con esto. Se ha notado un cambio de valores grande en la sociedad. La gente joven está más preocupada acerca de la marcha de la sociedad, el medio ambiente, la salud, la pobreza que hace cinco o diez años. En ese sentido, las cosas han progresado inesperadamente.
Debe serse optimista sobre el futuro. Se espera que Colombia siga manteniendo su ruta.

En todo caso el arquitecto debe ser el líder de las construcciones actuales, generando confianza y seguridad en el equipo de trabajo, eliminando todas las posibles fallas en la ejecución del proyecto, en el proceso de construcción y conociendo realmente el objetivo final que quiere el cliente. Debe procurar crear alianzas estratégicas buscando siempre los mayores beneficios y optimizar los recursos. Cabe resaltar que es de suma importancia estar siempre implementando procesos de mejoramiento y de estandarización de los mismos. Esto permitirá la correcta verificación y evaluación de los procesos. (Moreno, 2001)

Es sumamente necesario que las empresas de construcción y arquitectura cambien definitivamente los procesos actuales de construcción implantando procesos claros de gestión de calidad coherentes, para lograr un mejor funcionamiento de las obras, aumentando la productividad y buscando un mejor posicionamiento de la calidad en obra en nuestro país. (Moreno, 2001). 


\section{Referencias}

Alcoverro, J. (2013). Economía. Recuperado de http://es.wikipedia.org/ wiki/Econom\%C3\%ADa

Álvarez, C., Pabón, C., y Ortiz, J. F. (2010). Logística en Colombia: camino hacia la competitividad. Revista Económica Supuestos, 26-30.

Dane. (2005). Censo. Colombia.

Del Río, E. (1976). La trukulenta historia del kapitalismo. Mexico: Grijalbo.

Foster, R. (2011). Crisis económica y terror social. Recuperado de http:// experimentofilosofia.blogspot.com. co/2011/12/el-fin-del-capitalismo. html
Garay, L. (2004). Colombia: estructura industrial e internacionalización 19671996. Bogotá D.C.: Biblioteca Virtual del Banco de la República.

Moreno, B. (2001). La productividad y competitividad en obra. Bogotá: Universidad Piloto de Colombia.

Quesada, R. (2011). No estamos frente a una crisis económica, sino a una crisis cultural. Recuperado de http:// www.lanacion.com.ar/1059182-noestamos-frente-a-una-crisis-economica-sino-a-una-crisis-cultural

Restrepo, L. (2004). Interpretando a Porter. Bogota: Universidad del Rosario.

UM - Universidad de Manizales. (2006). Territorio y Competitividad. Manizales: Colciencias. 